\title{
EFEITOS DA $\beta$-POLINITRAÇÃO NA EFICIÊNCIA CATALÍTICA DE RUTENIOPORFIRINAS EM REAÇÕES DE OXIDAÇÃO DE CICLOEXANO
}

\author{
Christian Alexander Guimarães, Mirian Cristina dos Santos e Margarida de Moraes*
}

Departamento de Química, Universidade Federal de São Carlos, Rod. Washington Luis, km 235, 13565-905 São Carlos - SP

Recebido em 12/2/03; aceito em 8/9/03

\begin{abstract}
EFFECTS OF $\beta$-POLYNITRATION ON THE CATALYTIC EFFICIENCY OF RUTHENIUMPORPHYRINS IN CYCLOHEXANE OXIDATION. Rutheniumporphyrins, especially with several nitro groups in $\beta$-positions, were used in the cyclohexane oxidation in the presence of iodosylbenzene, hydrogen peroxide and sodium hypochlorite as oxygen donors, under mild conditions. The $\beta$-polynitrated complexes were able to promote the catalytic cyclohexane oxidation. They show an exceptionally high catalytic efficiency and resistance to attack by strong oxidizing agents. The cyclohexane oxidation was monitored by gas chromatography and the results showed that the $\beta$-polynitrated rutheniumporphyrins are better catalysts when compared to other complexes not $\beta$-polynitrated. In all cases, the 2-phenylsubstituted complexes were more efficient than 4-phenylsubstituted complexes. The importance of the ortho effect to oxidation was shown.
\end{abstract}

Keywords: polynitrated porphyrins; rutheniumporphyrins; oxidation.

\section{INTRODUÇÃO}

Rutenioporfirinas têm sido estudadas como catalisadores em diversos tipos de reações como, por exemplo, hidroxilação de cicloexano $^{1}$, ciclopropanações $^{2,3}$, reações de epoxidação ${ }^{4}$, detecção de oxigênio ${ }^{5}$, modelos para eficiência catalítica do citocromo $\mathrm{P} 450^{6}$ etc. Tal diversidade de reações catalisadas pode ser explicada, em parte, pela grande afinidade do complexo metaloporfirínico com átomos de oxigênio ${ }^{7}$ provindos, em muitos casos, de moléculas doadoras de oxigênio como, por exemplo, o iodosilbenzeno ${ }^{8,9}$, peróxido de hidrogênio ${ }^{10}$, monoperoxoftalatos ${ }^{11}$, hipoclorito de sódio ${ }^{12}$ etc. Outra explicação para a ampla possibilidade de utilização de rutenioporfirinas como catalisadores é a grande variedade nos estados de oxidação do rutênio nesses complexos. O rutênio pode sofrer variação entre $\mathrm{Ru}^{\mathrm{II}}$ e $\mathrm{Ru}^{\mathrm{VI}}$, sendo o estado $\mathrm{Ru}^{\mathrm{IV}}$ estabilizado na forma

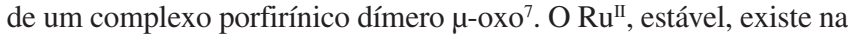
forma de $\mathrm{Ru}^{\mathrm{II}} \mathrm{P}(\mathrm{CO})(\mathrm{L})$ ou $\mathrm{Ru}^{\mathrm{II}} \mathrm{P}(\mathrm{L})_{2}$ onde $\mathrm{L}=\mathrm{CO}, \mathrm{ROH}, \mathrm{RCN}, \mathrm{R}_{3} \mathrm{P}$ ou piridina ${ }^{7}$.

A ligação reversível da molécula de oxigênio nas rutenioporfirinas, com o objetivo de mimetizar aspectos biológicos como os processos de oxigenação/oxidação, foi e continua sendo motivo de estudo de muitos pesquisadores ${ }^{13-17}$. Em solventes polares ou aprótipos como dimetilformamida, dimetilacetamida ou pirrol, o dioxigênio liga-se à rutênio-octaetilporfirina ou à meso-tetrafenilporfirinas do tipo da $\mathrm{Ru}$ (porfirina) $\mathrm{L}_{2}$ onde $\mathrm{L}=$ solvente, à temperatura ambiente e 1 atmosfera de pressão, fornecendo complexos do tipo $\mathrm{Ru}$ (porfirina) $\left(\mathrm{O}_{2}\right) \mathrm{L}$. Após algum tempo, a $20^{\circ} \mathrm{C}$, os complexos oxigenados decompõem-se em espécies $\mu$-oxo binucleares de rutênio. Inicialmente forma-se a espécie $\mathrm{Ru}(\mathrm{III})-\mathrm{O}-\mathrm{Ru}(\mathrm{III})$ que rapidamente se converte, na presença de traços de água, à espécie $\mu$-oxo binuclear de rutênio(IV) do tipo $[\mathrm{Ru} \text { (porfirina) }(\mathrm{OH})]_{2} \mathrm{O}^{16}$.

Muito se discute na literatura acerca de processos catalíticos envolvendo rutenioporfirinas e sua interação com átomos de oxigênio. Groves $^{13-16}$, dentre outros, afirma que a espécie dioxo-rutênio(VI) com tetramesitil ou tetradiclorofenilporfirina funciona como um efetivo catalisador para reações de oxidação de substratos orgânicos

*e-mail: marga@dq.ufscar.br como olefinas, alcanos, álcoois, fenóis e tioéteres onde o complexo transfere um átomo de oxigênio para o substrato realizando, portanto, a função de uma monooxigenase. Mlodnicka e James ${ }^{17}$ afirmam que estudaram vários processos de oxidação efetuados por complexos dioxo-rutênio(VI) e oxo-rutênio(IV) e que a espécie dioxo-rutênio(VI) é que seria a mais ativa em tais processos. Contudo, Oka e colaboradores $^{18}$, Bressan ${ }^{19}$, Chen $^{20}$, Morice $^{21}$, Hansen $^{22}$ e Guimarães $^{23}$, dentre outros, afirmam que a espécie oxo-rutênio(IV) é a espécie ativa nas reações de oxidação.

As rutenioporfirinas mostram-se catalisadores cada vez mais hábeis em reações de oxidação de substratos orgânicos ${ }^{12}$. Desde 1970 os trabalhos envolvendo estes complexos multiplicam-se intensamente, demonstrando suas habilidades como "complexos modelos" em substituir potencialmente enzimas que, na indústria, são utilizadas em processos de oxidação ${ }^{12,24,25}$. Sob esse aspecto, um catalisador ideal seria aquele que pudesse atender os interesses da indústria (alta seletividade, alta especificidade etc) e ao mesmo tempo ser estável a ponto de não sofrer oxidação, por exemplo. Na maioria dos casos, buscam-se tais propriedades através de metaloporfirinas $\beta$-funcionalizadas ${ }^{26}$.

A $\beta$-funcionalização de complexos meso-tetrafenilporfirinas é de grande interesse químico, já que muitas propriedades do macrociclo porfirínico podem ser alteradas por pequenas mudanças no anel ${ }^{26-29}$. À medida que substituintes são adicionados ao anel porfirínico, ocorrem distorções na simetria do complexo deixandoos com menor planaridade. Tal efeito pode ser de grande utilidade, por exemplo, em futuras aplicações desses compostos em terapia fotodinâmica cujos compostos, preferencialmente, não devem ter simetria muito alta para não sofrerem empacotamento.

A $\beta$-funcionalização de meso-tetrafenilporfirinas pode ser obtida a partir da polialogenação e da polinitração. A polinitração do macrociclo porfirínico é de interesse especial, já que os grupos nitro são facilmente convertidos em outros grupos funcionais ${ }^{27}$.

Nesse trabalho apresentamos alguns resultados comparativos entre rutenioporfirinas 2 e 4-nitrofenilsubstituídas e $\beta$-polinitropirrolsubstituídas como catalisadores na oxidação de cicloexano. Utilizou-se iodosilbenzeno, peróxido de hidrogênio e hipoclorito de sódio como doadores de oxigênio. 


\section{PARTE EXPERIMENTAL}

\section{Sínteses}

Síntese e purificação dos ligantes: tetraquis(5, 10, 15, 20-2nitrofenil)-21H, 23H-porfirina e tetraquis(5, 10, 15, 20-4-nitrofenil)21H, 23H-porfirina

As sínteses dos ligantes porfirínicos foram realizadas segundo adaptações da metodologia descrita por Lindsey ${ }^{30}$. Monitoraram-se as sínteses através de espectroscopia de absorção na região do ultravioleta-visível. Para isso, retiravam-se alíquotas de $0,1 \mathrm{~mL}$ que foram misturadas com alíquotas de $0,1 \mathrm{~mL}$ de 2,3-diciano-5,6-dicloro1,4-benzoquinona (solução estoque $10^{-2} \mathrm{~mol} \mathrm{~L}^{-1}$ ) em um balão volumétrico de $5 \mathrm{~mL}$, que foi completado, em seguida, com diclorometano tratado e seco. Após as sínteses, os ligantes foram purificados em coluna cromatográfica contendo sílica como fase estacionária e misturas de diclorometano/hexano como fase móvel, em várias proporções, dependendo do ligante a ser purificado.

Polinitração dos ligantes: tetraquis(5, 10, 15, 20-2-nitrofenil)-21H, $23 \mathrm{H}$-porfirina e tetraquis $(5,10,15,20-4$-nitrofenil)-21H, $23 \mathrm{H}$ porfirina

As sínteses dos ligantes porfirínicos $\beta$-nitropirrol-substituídos foram realizadas segundo adaptações da metodologia descrita por Bartoli $^{31}$. Os ligantes porfirínicos foram solubilizados em $100 \mathrm{~mL}$ de clorofórmio e $1 \mathrm{~mL}$ de metanol, de modo que a concentração da solução resultante fosse de $5 \times 10^{-3} \mathrm{~mol} \mathrm{~L}^{-1}$. Submeteu-se tal solução a resfriamento por meio de banho de gelo. Foram gotejados 100 equivalentes de ácido nítrico concentrado durante $3 \mathrm{~h}$, deixando-se então a reação sob agitação mecânica até completar 24 h de seu início. Ao término deste período o solvente foi evaporado, obtendo-se então o ligante polinitrado, impuro, na forma de pó. As reações de polinitração foram monitoradas por espectrometria de absorção na região do ultravioleta-visível (deslocamento da banda Soret para maiores valores de comprimento de onda e desaparecimento de três das quatro bandas Q). Após a síntese, os ligantes foram purificados por cromatografia em coluna usando-se óxido de silício como fase estacionária e uma mistura de diclorometano/metanol na proporção de 10:1 como fase móvel.

Síntese e purificação dos complexos: [(carbonil)(2-nitrofenil) porfirinato]rutênio(II), [(carbonil)(4-nitrofenil)porfirinato] rutênio(II), [(carbonil)(2-nitrofenil) $\beta$-polinitroporfirinato] rutênio(II), [(carbonil)(4-nitrofenil) $\beta$-polinitroporfirinato] rutênio(II)

O rutênio foi inserido nos ligantes porfirínicos via reação de tricloreto de rutênio com o respectivo ligante porfirínico dissolvido em 2-metoxietanol, sob fluxo constante de monóxido de carbono (adaptações da metodologia descrita por Collman ${ }^{32}$ ). As reações de metalação também foram monitoradas por espectroscopia de absorção na região do ultravioleta-visível. Após as sínteses, os complexos foram purificados em coluna cromatográfica contendo óxido de alumínio neutro como fase estacionária e diclorometano/hexano/acetona, em várias proporções, como fase móvel.

\section{Síntese e padronização do iodosilbenzeno (doador de oxigênio)}

O iodosilbenzeno foi obtido por hidrólise alcalina do diacetato de iodobenzeno, segundo a metodologia descrita por Sharefkin ${ }^{33}$, de acordo com a equação química mostrada na Figura 1.

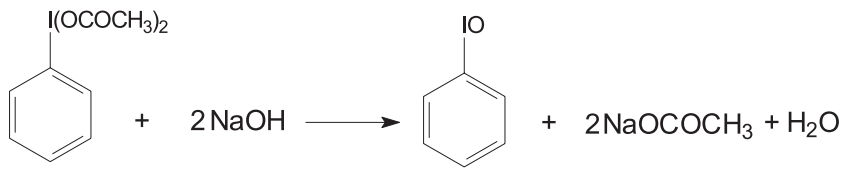

Figura 1. Equação química que representa a síntese do iodosilbenzeno

Após a síntese e antes de cada utilização, o iodosilbenzeno foi padronizado por iodometria, segundo a metodologia descrita por Lucas $^{34}$.

\section{Reação de oxidação do cicloexano}

As reações de oxidação do cicloexano foram realizadas em frascos de vidro de $3 \mathrm{~mL}$ de capacidade, com tampa com septo de teflon interfaceado com silicone. Para cada reação catalisada envolvendo o iodosilbenzeno como doador de oxigênio, foram pesados no próprio frasco o catalisador porfirínico e o doador de oxigênio. Em seguida, foi colocada uma barra magnética e o frasco foi vedado. Utilizandose agulhas e seringas ligadas a uma linha de gás, seu conteúdo foi desaerado por 40 min com argônio puro e seco. Após esse tempo foram adicionados, usando-se microsseringa, diclorometano $(1,4 \mathrm{~mL})$ e cicloexano (1,5 $\mathrm{mL})$, ambos puros e previamente desaerados com argônio. Agitou-se o conteúdo do frasco por ultra-som ou mecanicamente ou ambos os sistemas.

Para cada reação catalisada envolvendo doadores de oxigênio líquidos, peróxido de hidrogênio e hipoclorito de sódio, pesou-se, no próprio frasco, o catalisador porfirínico. Em seguida, foi colocada uma barra magnética e o frasco foi vedado. Utilizando-se agulhas e seringas ligadas a uma linha de gás, seu conteúdo foi desaerado por 40 min com argônio puro e seco. Após esse tempo foram adicionados diclorometano $(1,4 \mathrm{~mL})$ e cicloexano $(1,5 \mathrm{~mL})$ ambos puros e previamente desaerados com argônio. $\mathrm{Na}$ seqüência, iniciou-se agitação da reação por ultra-som ou mecanicamente com a concomitante adição lenta, através de microsseringa, do doador de oxigênio no estado líquido, desaerado previamente por $10 \mathrm{~min}$ com argônio puro e seco. Em todos os casos os doadores de oxigênio foram padronizados antes do uso e as reações foram mantidas na ausência de luz, oxigênio e à temperatura ambiente.

Os produtos de oxidação do cicloexano foram analisados por cromatografia a gás, usando-se o método do padrão interno e o rendimento dos produtos foi determinado com base na quantidade de doador de oxigênio utilizado.

\section{Estudos de recuperação dos catalisadores}

Inicialmente foram determinados os coeficientes de absortividade molar ( $\varepsilon$ ) dos complexos rutenioporfirínicos. Isso foi feito a partir de medidas da intensidade das bandas Soret (bandas mais intensas do espectro eletrônico). Prepararam-se soluções dos complexos, em diclorometano, com concentração variando de $10^{-1}$ a $10^{-6} \mathrm{~mol} \mathrm{~L}^{-1} \mathrm{e}$ obtiveram-se os respectivos espectros eletrônicos. Usando-se a lei de Lambert-Beer determinaram-se os $\varepsilon$ das rutenioporfirinas.

Após a análise dos produtos das reações de oxidação, os respectivos espectros eletrônicos dessas soluções contendo os complexos porfirínicos foram obtidos. A partir dos valores da absorção da banda Soret dos complexos presentes nessas soluções com volumes e quantidades iniciais conhecidos, calculou-se, usando o valor de absortividade molar determinado para cada complexo, a porcentagem de complexo (catalisador) ainda presente depois de realizada a catálise. 


\section{RESULTADOS}

\section{Caracterização dos compostos sintetizados}

A Tabela 1 apresenta os dados que caracterizam os ligantes porfirínicos sintetizados e purificados.

Analisando-se e comparando-se os valores da Tabela 1 com respeito às atribuições na região do infravermelho observou-se que, após a polinitração dos ligantes porfirínicos, ocorreu o desaparecimento dos picos que correspondem à deformação $-\mathrm{C}-\mathrm{H}$ pirrol. Esse dado pode indicar que os hidrogênios pirrólicos podem ter sido substituídos por grupos nitro. Os dados de análise elementar demonstram que, provavelmente, nem todos os hidrogênios pirrólicos foram substituídos, uma vez que tais dados são condizentes com apenas cinco grupos nitro nas posições $\beta$-pirrólicas, para o tetraquis $[(5,10,15$, 20 -2-nitrofenil)- $\beta$-polinitro]-21H, $23 \mathrm{H}$-porfirina e sete grupos nitro nas posições $\beta$-pirrólicas para o tetraquis $[(5,10,15,20$-4-nitrofenil)$\beta$-polinitro]-21H, 23H-porfirina. Isso leva a crer que a $\beta$-polinitração é limitada estericamente, uma vez que o ligante 2-substituído teve menor polinitração. Os dados obtidos com a espectroscopia eletrônica na região do ultravioleta-visível demonstram uma nítida alteração de simetria da molécula ao introduzir grupos nitro nas posições $\beta$-pirrólicas.

A Tabela 2 apresenta os dados que caracterizam os complexos porfirínicos sintetizados e purificados.

O aparecimento de uma banda na região de $1940 \mathrm{~cm}^{-1}$ a 1990 $\mathrm{cm}^{-1}$ (dados de espectroscopia de absorção na região do infravermelho) indica a coordenação de monóxido de carbono ao rutênio do centro do anel porfirínico. Essa informação, associada às mudanças nas absorções do espectro eletrônico e aos dados de análise elementar, é um importante indício que a metalação ocorreu.
Avaliação da eficiência catalítica dos complexos sintetizados

A reação estudada é representada na Figura 2.

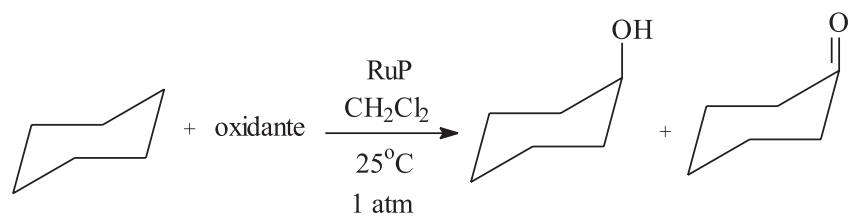

Figura 2. Esquema da reação catalisada estudada onde $R u P=$ complexo rutenioporfirínico

Na Tabela 3 são mostrados os valores dos rendimentos de cicloexanol (c-ol) e cicloexanona (c-ona) obtidos para a reação catalisada de oxidação do cicloexano com iodosilbenzeno em atmosfera inerte, utilizando os complexos rutenioporfirínicos de segunda e terceira geração. Os rendimentos foram calculados com base na quantidade de doador de oxigênio utilizado. Também se pode observar o efeito do substituinte nas posições 2 e 4-fenil no rendimento dos produtos.

A análise dos dados da Tabela 3 mostra que a relação entre o catalisador e o doador de oxigênio influencia nos rendimentos dos produtos. É bastante notório que o aumento da proporção de oxidante na reação desfavorece o processo catalítico. Provavelmente ocorre a inibição da formação do intermediário de alta valência levando à formação de iodoxibenzeno e regenerando o catalisador original ${ }^{19}$. Também é interessante observar, analisando os dados da Tabela 3, que para os complexos polinitrados a melhor relação molar entre catalisador/doador de oxigênio é de 1:5 (mol/mol) enquanto que para os não polinitrados essa é de 1:10 ( $\mathrm{mol} / \mathrm{mol})$. Isso confirma o fato de

Tabela 1. Dados experimentais que caracterizam todos os ligantes porfirínicos sintetizados

\begin{tabular}{|c|c|c|c|c|c|c|}
\hline \multirow[t]{2}{*}{ Ligante } & \multicolumn{2}{|c|}{ Ultravioleta-visível } & \multicolumn{2}{|c|}{ Análise elementar CHN } & \multicolumn{2}{|c|}{ Infravermelho } \\
\hline & $\begin{array}{c}\text { Banda } \\
\text { Soret/nm }\end{array}$ & $\begin{array}{c}\text { Bandas } \\
\text { Q/nm }\end{array}$ & calculado/\% & experimental $/ \%$ & $\begin{array}{c}\text { Principais } \\
\text { atribuições } \\
\text { tentativas }^{32,35-39}\end{array}$ & $\begin{array}{l}\text { Número de } \\
\text { onda/ } \mathrm{cm}^{-1}\end{array}$ \\
\hline \multirow[t]{4}{*}{1} & 422 & 520 & $C=66,5$ & $C=67,3$ & $\mathrm{NO}_{2}$ angular & 784 \\
\hline & & 555 & $\mathrm{H}=3,30$ & $\mathrm{H}=3,51$ & $\mathrm{NH}$ angular & 3423 \\
\hline & & 590 & $\mathrm{~N}=14,1$ & $\mathrm{~N}=14,2$ & $\mathrm{C}-\mathrm{N}_{\text {anel }}$ & 1362 \\
\hline & & 664 & & & $\mathrm{C}-\mathrm{H}_{\text {pirrol }}$ & 1421 \\
\hline \multirow[t]{5}{*}{2} & 450 & 510 & $\mathrm{C}=51,8$ & $\mathrm{C}=51,2$ & $\mathrm{NO}_{2}$ angular & 711 \\
\hline & & & $\mathrm{H}=2,08$ & $\mathrm{H}=1,90$ & $\mathrm{NH}$ axial simétrica & 1445 \\
\hline & & & $\mathrm{N}=17,86$ & $\mathrm{~N}=17,4$ & $\mathrm{C}-\mathrm{N}_{\mathrm{anel}}$ & 1278 \\
\hline & & & & & C-H $\mathrm{H}_{\text {pirrol }}$ & $\longrightarrow$ \\
\hline & & & & & $\mathrm{C}_{\text {pirrol }}-\mathrm{NO}_{2}$ & 1343 \\
\hline \multirow[t]{4}{*}{3} & 424 & 514 & $C=66,5$ & $C=68,0$ & $\mathrm{NO}_{2}$ angular & 709 \\
\hline & & 546 & $\mathrm{H}=3,33$ & $\mathrm{H}=2,91$ & $\mathrm{NH}$ angular & 3393 \\
\hline & & 586 & $\mathrm{~N}=14,1$ & $\mathrm{~N}=13,9$ & $\mathrm{C}-\mathrm{N}_{\text {anel }}$ & 1313 \\
\hline & & 666 & & & C-Hpirrol & 1411 \\
\hline \multirow[t]{5}{*}{4} & 440 & 510 & $C=47,6$ & $C=46,9$ & $\mathrm{NO}_{2}$ angular & 710 \\
\hline & & & $\mathrm{H}=1,13$ & $\mathrm{H}=1,56$ & $\mathrm{NH}$ axial simétrica & 1596 \\
\hline & & & $\mathrm{N}=18,93$ & $\mathrm{~N}=19,3$ & $\mathrm{C}-\mathrm{N}_{\text {anel }}$ & 1289 \\
\hline & & & & & $\mathrm{C}-\mathrm{H}_{\text {pirrol }}$ & $\longrightarrow$ \\
\hline & & & & & $\mathrm{C}_{\text {pirrol }}-\mathrm{NO}_{2}$ & 1344 \\
\hline
\end{tabular}

$\mathbf{1}=$ tetraquis $(5,10,15,20$-2-nitrofenil)-21H, 23H-porfirina; 2 = tetraquis $[(5,10,15,20$-2-nitrofenil) $\beta$-pentanitro $]$ - $21 \mathrm{H}, 23 \mathrm{H}$-porfirina; $\mathbf{3}=$ tetraquis $(5,10,15,20-4$-nitrofenil)-21H, 23H-porfirina e $\mathbf{4}=$ tetraquis[ $[(5,10,15,20-4$-nitrofenil) $\beta$-heptanitro]-21H, $23 \mathrm{H}$-porfirina 
Tabela 2. Dados experimentais que caracterizam todos os complexos porfirínicos sintetizados

\begin{tabular}{|c|c|c|c|c|c|c|}
\hline \multirow[t]{2}{*}{ Complexo } & \multicolumn{2}{|c|}{ Ultravioleta-visível } & \multicolumn{2}{|c|}{ Análise elementar CHN } & \multicolumn{2}{|c|}{ Infravermelho } \\
\hline & $\begin{array}{c}\text { Banda } \\
\text { Soret/nm }\end{array}$ & $\begin{array}{c}\text { Bandas } \\
\text { Q/nm }\end{array}$ & calculado/\% & experimental $/ \%$ & $\begin{array}{c}\text { Principais } \\
\text { atribuições } \\
\text { tentativas }^{32,35-39}\end{array}$ & $\begin{array}{l}\text { Número de } \\
\text { onda/ } \mathrm{cm}^{-1}\end{array}$ \\
\hline 1 & 420 & $\begin{array}{l}520 \\
583\end{array}$ & $\begin{array}{l}\mathrm{C}=58,6 \\
\mathrm{H}=2,62 \\
\mathrm{~N}=12,2\end{array}$ & $\begin{array}{l}\mathrm{C}=58,1 \\
\mathrm{H}=2,78 \\
\mathrm{~N}=12,8\end{array}$ & $\begin{array}{c}\mathrm{NO}_{2} \text { simétrica } \\
\mathrm{NO}_{2} \text { angular } \\
\mathrm{CO} \text { coordenado }\end{array}$ & $\begin{array}{c}1427 \\
786 \\
1956\end{array}$ \\
\hline 2 & 410 & 550 & $\begin{array}{l}\mathrm{C}=43,7 \\
\mathrm{H}=1,39 \\
\mathrm{~N}=17,0\end{array}$ & $\begin{array}{l}\mathrm{C}=43,1 \\
\mathrm{H}=1,47 \\
\mathrm{~N}=17,3\end{array}$ & $\begin{array}{c}\mathrm{NO}_{2} \text { simétrica } \\
\mathrm{NO}_{2} \text { angular } \\
\mathrm{C}_{\text {pirrol }}-\mathrm{NO}_{2} \\
\mathrm{CO} \text { coordenado }\end{array}$ & $\begin{array}{l}1457 \\
765 \\
1261 \\
1961\end{array}$ \\
\hline 3 & 420 & $\begin{array}{l}518 \\
584\end{array}$ & $\begin{array}{l}\mathrm{C}=58,6 \\
\mathrm{H}=2,62 \\
\mathrm{~N}=12,2\end{array}$ & $\begin{array}{l}\mathrm{C}=58,3 \\
\mathrm{H}=2,66 \\
\mathrm{~N}=12,5\end{array}$ & $\begin{array}{c}\mathrm{NO}_{2} \text { simétrica } \\
\mathrm{NO}_{2} \text { angular } \\
\mathrm{CO} \text { coordenado }\end{array}$ & $\begin{array}{l}1422 \\
- \\
1949\end{array}$ \\
\hline 4 & 430 & 552 & $\begin{array}{l}\mathrm{C}=47,0 \\
\mathrm{H}=1,63 \\
\mathrm{~N}=15,5\end{array}$ & $\begin{array}{l}\mathrm{C}=46,6 \\
\mathrm{H}=1,95 \\
\mathrm{~N}=15,8\end{array}$ & $\begin{array}{c}\mathrm{NO}_{2} \text { simétrica } \\
\mathrm{NO}_{2} \text { angular } \\
\mathrm{C}_{\text {pirrol }}-\mathrm{NO}_{2} \\
\text { CO coordenado }\end{array}$ & $\begin{array}{l}1455 \\
-- \\
1245 \\
1981\end{array}$ \\
\hline
\end{tabular}

$\mathbf{1}=$ (carbonil)[tetraquis $(5,10,15,20-2$-nitrofenil)porfirinato]rutênio(II); $\mathbf{2}=$ (carbonil)[tetraquis(5,10,15,20-2-nitrofenil- $\beta$ pentanitro)porfirinato]rutênio(II); 3 = (carbonil)[tetraquis (5,10,15,20-4-nitrofenil)porfirinato]rutênio(II) e $\mathbf{4}=($ carbonil) [tetraquis(5,10,15,204-nitrofenil- $\beta$-heptanitro)porfirinato]rutênio(II)

Tabela 3. Resultados da oxidação do cicloexano por iodosilbenzeno usando-se os catalisadores de segunda e terceira geração para diferentes relações catalisador:doador de oxigênio

\begin{tabular}{|c|c|c|c|c|c|c|c|c|}
\hline \multirow{3}{*}{$\begin{array}{c}\text { catalisador/ } \\
\text { doador de } \\
\text { oxigênio }(\mathrm{mol} / \mathrm{mol})\end{array}$} & \multicolumn{8}{|c|}{ Produtos formados } \\
\hline & \multicolumn{2}{|c|}{ Complexo 1} & \multicolumn{2}{|c|}{ Complexo 2} & \multicolumn{2}{|c|}{ Complexo 3} & \multicolumn{2}{|c|}{ Complexo 4} \\
\hline & $\mathrm{c}-\mathrm{ol} / \%$ & c-ona/\% & $\mathrm{c}-\mathrm{ol} / \%$ & c-ona/\% & $\mathrm{c}-\mathrm{ol} / \%$ & c-ona/\% & $\mathrm{c}-\mathrm{ol} / \%$ & c-ona $/ \%$ \\
\hline $1: 5$ & 31 & 28 & 85 & 84 & 6 & 1 & 17 & 17 \\
\hline $1: 10$ & 45 & 30 & 86 & 70 & 8 & 2 & 8 & 7 \\
\hline $1: 15$ & 29 & 25 & 35 & 32 & 4 & 1 & 5 & 4 \\
\hline
\end{tabular}

Utilizou-se 1 minuto de agitação por ultra-som e $30 \mathrm{mi}$ de agitação mecânica. A concentração de catalisador foi de aproximadamente $10^{-4} \mathrm{~mol} \mathrm{~L}^{-1}$. complexo 1 = (carbonil)[tetraquis(5,10,15,20-2-nitrofenil)porfirinato]rutênio(II); complexo $2=($ carbonil)[tetraquis $(5,10,15,20$ 2-nitrofenil- $\beta$-pentanitro)porfirinato] rutênio(II); complexo 3 = (carbonil)[tetraquis(5,10,15,20-4-nitrofenil)porfirinato]rutênio(II) e complexo $4=($ carbonil) [tetraquis $(5,10,15,20-4$-nitrofenil- $\beta$-heptanitro)porfirinato]rutênio(II)

que porfirinas polinitradas, apesar de serem consideradas mais estáveis em termos de destruição oxidativa durante a catálise, provavelmente levam mais facilmente o iodosilbenzeno a iodoxibenzeno, como sugerido por Bressan ${ }^{19}$. Ele sugere que o iodosilbenzeno, durante o processo catalítico, por ação de um intermediário do catalisador porfirínico, $\mathrm{Ru}(\mathrm{IV}) \mathrm{P}=\mathrm{O}$, se oxida regenerando o catalisador original e consumindo o doador de oxigênio. Isso é ilustrado na Figura 3.

Comparando-se os rendimentos dos produtos entre as porfirinas de segunda geração, percebe-se que os complexos 2-nitro-substituídos levam a um maior rendimento dos produtos de oxidação. Isso pode ser explicado pelo fato dos grupos nitro na posição 2-fenil levarem a uma maior distorção do anel porfirínico, menor simetria e maior exposição do centro metálico. Isso gera a estabilização de intermediários de alta valência, o que leva a maiores rendimentos e seletividade dos produtos da reação catalisada. Por consequiência, nos complexos 4-nitro-substituídos pode ocorrer o inverso. Com menor estabilidade, os intermediários de alta valência podem ser convertidos para intermediários de valência menor e assim colaborar para a oxidação do próprio doador de oxigênio, pois nesse caso, a cinética da reação deverá ser favorecida. Para os complexos polinitrados, especialmente o 2-nitropentanitro, observa-se maior contribuição no que se refere à formação de produtos. Isso decorre da maior distorção da simetria do anel porfirínico causada pela interação dos grupos 2-nitrofenil com os grupos $\beta$-nitropirrol.

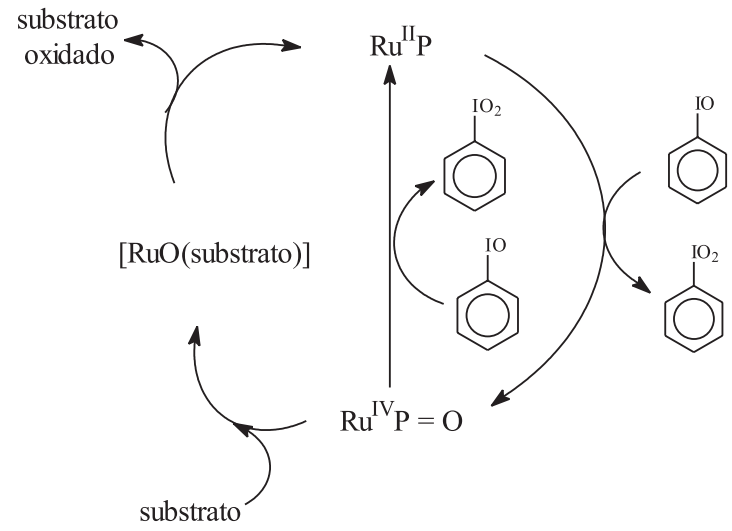

Figura 3. Mecanismos propostos para a catálise oxidativa usando rutenioporfirinas. Em especial a formação da espécie oxidada do doador de oxigênio. $R u P=$ complexo rutenioporfirínico 
É importante observar nos resultados, a formação preferencial, em alguns casos, de cicloexanol. Isso pode ser explicado pela possibilidade da reação catalisada envolver, pelo menos em parte, um mecanismo radicalar $^{23,35-43}$. Ciuffi ${ }^{42}$ propõe em sua tese de doutoramento que radicais $\mathrm{R}^{\circ}$, provavelmente originados do solvente da reação, podem reagir com oxigênio molecular (podendo existir na forma de traços no meio reacional) ou até mesmo com o doador de oxigênio, com subseqüente intermédio do catalisador porfirínico, formando a espécie $\mathrm{RO}^{\circ}$, que interage com o substrato formando o álcool correspondente ao substrato.

Baseado no mecanismo proposto por $\mathrm{Ciuffi}^{42}$ para ferroporfirinas, pode-se propor um mecanismo envolvendo os resultados obtidos com rutenioporfirinas. Tal mecanismo envolveria provavelmente a formação de intermediários $\mathrm{Ru}^{\mathrm{IV}}$, por exemplo, como sugerido por Meunier ${ }^{12}$, Bressan $^{19}$ ou Sheldon ${ }^{41}$. A Figura 4 mostra uma proposta de mecanismo para explicar a formação preferencial de cicloexanol, quando se utilizam rutenioporfirinas como catalisadores.

Os estudos de recuperação dos catalisadores demonstraram que os complexos $\beta$-nitro-substituídos têm maior porcentagem de recuperação mesmo após um tempo de reação considerado longo (144 h). Tais dados são apresentados na Tabela 4.

Os resultados apresentados na Tabela 4 evidenciam que, no tempo de reação estudado para a obtenção dos resultados de oxidação apresentados na Tabela 3, a destruição dos catalisadores é muito pequena, porém, para tempos maiores de reação, 24 h ou mais, os complexos polinitrados apresentam maior resistência à destruição.

O tipo e tempo de agitação do sistema catalisado também se mostraram importantes no que se refere ao rendimento dos produtos. Na Tabela 5 encontram-se os rendimentos de cicloexanol e cicloexanona, a partir da reação do cicloexano com iodosilbenzeno catalisada pelos complexos polinitrados, enfatizando o efeito do tipo de agitação nesses sistemas.

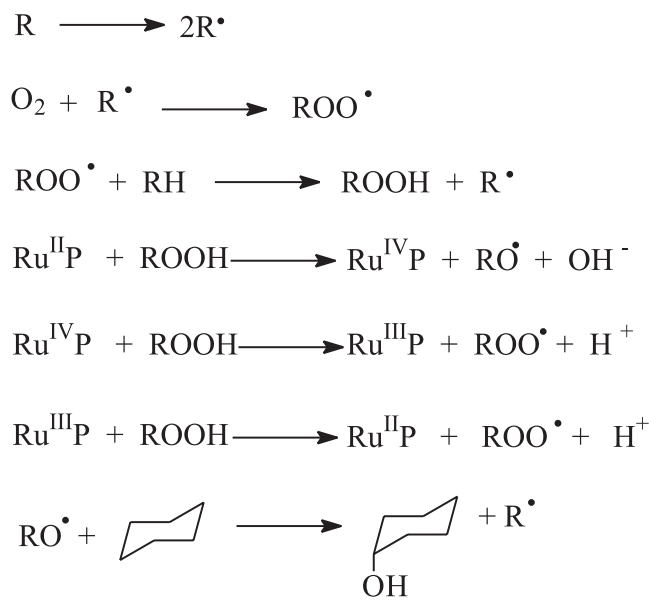

Figura 4. Proposta de mecanismo radicalar de formação de cicloexanol onde $R u P=$ rutenioporfirina

Como pode ser observado na Tabela 5, o tipo de agitação exerce bastante influência sobre os rendimentos obtidos. Em geral, observou-se pouca alteração nas quantidades de produtos formados quando se utilizou somente a agitação mecânica em diferentes tempos de agitação. Nessas condições o complexo 2-nitrofenil- $\beta$-pentanitro apresenta melhores resultados em termos de formação de produtos. Isso pode ser explicado pelo fato desse complexo possuir maior distorção estrutural, provocada pelo efeito estéreo dos grupos nitro em posição orto-fenil interagindo com os grupos nitro da posição beta pirrólica. Isso faz com que o anel porfirínico distorça mais e o metal se desloque mais do centro desse anel, facilitando a aproximação do substrato e do doador de oxigênio ao centro metálico.

Quando somente o ultra-som foi utilizado como forma de agita-

Tabela 4. Dados de recuperação dos complexos utilizados nas catálises

\begin{tabular}{lcccc}
\hline & \multicolumn{4}{c}{ Porcentagem de catalisador } \\
& Complexo 1 & Complexo 2 & Complexo 3 & Complexo 4 \\
\hline Após término da agitação da reação & 99 & 99 & 98 & 99 \\
12 h após término da agitação da reação & 85 & 98 & 73 & 80 \\
24 h após término da agitação da reação & 71 & 92 & 64 & 76 \\
144 h após término da agitação da reação & 14 & 84 & 8 & 67 \\
\hline
\end{tabular}

Agitaram-se as reações por 1 min de ultra-som e 30 min de agitação mecânica. A relação catalisador/doador de oxigênio foi 1:5 (mol:mol) para os complexos polinitrados e 1:10 (mol:mol) para os não polinitrados. A concentração de catalisador foi de aproximadamente $10^{-4}$ mol $\mathrm{L}^{-1}$. complexo 1 = (carbonil)[tetraquis (5,10,15,20-2-nitrofenil)porfirinato]rutênio(II); e complexo 2 = (carbonil)[tetraquis(5,10,15,20-2-nitrofenil$\beta$-pentanitro)porfirinato]rutênio(II); complexo $3=($ carbonil) [tetraquis(5,10,15,20-4-nitrofenil)porfirinato]rutênio(II) e complexo 4 = (carbonil)[tetraquis $(5,10,15,20$-4-nitrofenil- $\beta$-heptanitro)porfirinato]rutênio(II).

Tabela 5. Efeito do tipo de agitação na reação de oxidação do cicloexano por iodosilbenzeno catalisada pelos complexos $\beta$-substituídos

\begin{tabular}{|c|c|c|c|c|c|c|c|c|}
\hline \multirow{3}{*}{$\begin{array}{c}\text { Tempo de } \\
\text { agitação/min }\end{array}$} & \multicolumn{4}{|c|}{ Complexo 1} & \multicolumn{4}{|c|}{ Complexo 2} \\
\hline & \multicolumn{2}{|c|}{ Agitação por ultra-som } & \multicolumn{2}{|c|}{ Agitação mecânica } & \multicolumn{2}{|c|}{ Agitação por ultra-som } & \multicolumn{2}{|c|}{ Agitação mecânica } \\
\hline & $\mathrm{c}-\mathrm{ol} / \%$ & c-ona/\% & $\mathrm{c}-\mathrm{ol} / \%$ & c-ona/ $\%$ & $\mathrm{c}-\mathrm{ol} / \%$ & c-ona/\% & $\mathrm{c}-\mathrm{ol} / \%$ & c-ona/\% \\
\hline 1 & 27 & 26 & 9 & 7 & 8 & 6 & - & - \\
\hline 5 & 54 & 53 & 57 & 54 & 14 & 11 & 9 & 7 \\
\hline 10 & 74 & 73 & 57 & 54 & 16 & 14 & 12 & 10 \\
\hline 30 & 75 & 75 & 57 & 54 & 17 & 15 & 13 & 12 \\
\hline 50 & 76 & 75 & 57 & 53 & 18 & 18 & 15 & 13 \\
\hline
\end{tabular}

A relação complexo/doador de oxigênio foi 1:5 (mol:mol). A concentração de catalisador foi de aproximadamente $10^{-4}$ mol $\mathrm{L}^{-1}$. complexo $1=$ (carbonil)[tetraquis(5,10,15,20-2-nitrofenil- $\beta$-pentanitro)porfirinato]rutênio(II) e complexo 2 = (carbonil)[tetraquis(5,10,15,20-4-nitrofenil$\beta$-heptanitro)porfirinato]rutênio(II) 
ção da reação, observou-se rendimentos melhores de produtos, se comparados àqueles obtidos com a agitação mecânica. Isso sugere um mecanismo de reação radicalar.

Pode-se observar em alguns casos, nas Tabelas 3 e 5, que a soma dos rendimentos de cicloexanol e cicloexanona supera $100 \%$. Tal fato pode ser explicado pela contribuição de oxigênio molecular que pode existir na solução e que pode ser consumido durante a reação catalisada. Embora o meio de reação tenha sido desaerado previamente é possível que nem todo oxigênio tenha sido completamente removido do frasco de reação, permanecendo nos interstícios dos sólidos, sendo transferido para a reação após a dissolução desses. Nesse caso o oxigênio pode ter participado da reação levando a um aumento da porcentagem de produtos.

A influência de diferentes doadores de oxigênio nas reações catalisadas pelos complexos sintetizados também foi estudada. Os resultados são apresentados na Tabela 6 . A fim de facilitar a comparação entre os resultados, os dados obtidos com o iodosilbenzeno foram também incluídos nessa tabela.

Como pode ser observado na Tabela 6 , o tipo de doador de oxigênio não provoca mudanças significativas nos rendimentos dos produtos quando comparados com o iodosilbenzeno. O que provavelmente ocorre é que, quando se utiliza um doador de oxigênio com características oxidantes mais pronunciadas este passa a atuar de forma mais agressiva podendo levar a uma destruição maior do catalisador. Outra possibilidade é que a decomposição do doador de oxigênio, principalmente o peróxido de hidrogênio, ocorra mais rápido do que o tempo que ele levaria para participar da reação. Para constatar tais fatos, foram realizados estudos de recuperação do catalisador após as reações com o iodosilbenzeno, hipoclorito de sódio e peróxido de hidrogênio. A Tabela 7 mostra os valores da porcentagem de recuperação dos catalisadores, logo após a agitação da reação, quando se utilizou diferentes doadores de oxigênio.

Os dados da Tabela 7 demonstram que realmente o poder oxidante do doador de oxigênio é predominante. Pode-se observar baixa recuperação dos catalisadores para o peróxido de hidrogênio e alta para o iodosilbenzeno. Isso demonstra que, quando se utiliza o peróxido como doador de oxigênio, ocorre uma maior destruição do catalisador mesmo para os complexos $\beta$-nitro-substituídos. Também se pode sugerir a possibilidade de decomposição do doador de oxigênio antes dele ser utilizado na reação de oxidação.

\section{CONCLUSÕES}

Os resultados obtidos indicam que porfirinas polinitradas, apesar de serem consideradas mais estáveis em termos de destruição oxidativa durante a catálise, provavelmente levam mais facilmente o iodosilbenzeno a iodoxibenzeno.

Também foi constatado que a forma de agitação das reações exerce bastante influência sobre os rendimentos obtidos. Em geral, observou-se pouca alteração nas quantidades de produtos formados quando se utilizou somente a agitação mecânica em diferentes tempos de agitação. A utilização do ultra-som por curto período de tempo leva a rendimentos maiores do que se utilizado por tempos longos. Provavelmente nesse último caso ocorra destruição dos complexos porfirínicos. Nessas condições observou-se grande importância do efeito orto nos complexos. Observou-se maiores rendimentos de pro-

Tabela 6. Oxidação do cicloexano com diferentes doadores de oxigênio

\begin{tabular}{|c|c|c|c|c|c|c|c|c|c|}
\hline \multirow{2}{*}{$\begin{array}{l}\text { Doador de } \\
\text { oxigênio }\end{array}$} & \multirow{2}{*}{$\begin{array}{c}\text { Tempo e tipo } \\
\text { de agitação/ min }\end{array}$} & \multicolumn{2}{|c|}{ Complexo 1} & \multicolumn{2}{|c|}{ Complexo 2} & \multicolumn{2}{|c|}{ Complexo 3} & \multicolumn{2}{|c|}{ Complexo 4} \\
\hline & & $\mathrm{c}-\mathrm{ol} / \%$ & c-ona/\% & $\mathrm{c}-\mathrm{ol} / \%$ & c-ona/\% & $\mathrm{c}-\mathrm{ol} / \%$ & c-ona/\% & $\mathrm{c}-\mathrm{ol} / \%$ & c-ona/\% \\
\hline $\mathrm{PhIO}$ & 1 min ultra-som & 19 & 11 & 27 & 26 & 4 & 2 & 13 & 14 \\
\hline $\mathrm{NaOCl}$ & & 28 & 25 & 29 & 27 & 6 & 4 & 15 & 17 \\
\hline $\mathrm{H}_{2} \mathrm{O}_{2}$ & & 23 & 22 & 22 & 20 & 5 & Traços & 9 & 8 \\
\hline $\mathrm{PhIO}$ & 30 min mecânica & 40 & 21 & 57 & 54 & 6 & 3 & 13 & 15 \\
\hline $\mathrm{NaOCl}$ & & 37 & 29 & 60 & 58 & 7 & 5 & 14 & 15 \\
\hline $\mathrm{H}_{2} \mathrm{O}_{2}$ & & 28 & 26 & 56 & 53 & 5 & 2 & 12 & 9 \\
\hline $\mathrm{PhIO}$ & Mista* & 45 & 30 & 85 & 84 & 8 & 2 & 17 & 17 \\
\hline $\mathrm{NaOCl}$ & & 47 & 32 & 87 & 85 & 10 & 5 & 18 & 16 \\
\hline $\mathrm{H}_{2} \mathrm{O}_{2}$ & & 40 & 22 & 82 & 83 & 7 & 1 & 14 & 13 \\
\hline
\end{tabular}

A relação complexo/doador de oxigênio foi 1:5 (mol:mol) para os complexos polinitrados e 1:10 (mol:mol) para os demais. A concentração de catalisador foi de aproximadamente $10^{-4} \mathrm{~mol} \mathrm{~L}^{-1}$. complexo 1 = (carbonil)[tetraquis(5,10,15,20-2-nitrofenil)porfirinato]rutênio(II); complexo $2=($ carbonil) [tetraquis $(5,10,15,20-2$-nitrofenil- $\beta$-pentanitro)porfirinato]rutênio(II); complexo $3=($ carbonil) [tetraquis $(5,10,15,20-4-$ nitrofenil)porfirinato]rutênio(II) e complexo $4=$ (carbonil) [tetraquis $(5,10,15,20-4$-nitrofenil- $\beta$-heptanitro)porfirinato]rutênio(II). *mista $=$ 1 min ultra-som e 30 min agitação mecânica.

Tabela 7. Estudos de recuperação dos catalisadores após reação catalisada na presença de diferentes doadores de oxigênio

\begin{tabular}{lcccc}
\hline Doador de oxigênio & \multicolumn{4}{c}{ Recuperação do catalisador\% } \\
& Complexo 1 & Complexo 2 & Complexo 3 & Complexo 4 \\
\hline Iodosilbenzeno & 99 & 99 & 98 & 99 \\
Hipoclorito de sódio & 85 & 88 & 83 & 87 \\
Peróxido de hidrogênio & 37 & 43 & 34 & 36 \\
\hline
\end{tabular}

As condições das reações foram: relação molar complexo/doador de oxigênio 1:5 (complexos polinitrados) e 1:10 (complexos não polinitrados). Os dados foram obtidos logo após a agitação da reação catalisada (agitação: 1 min ultra-som e 30 min mecânica). complexo 1 = (carbonil)[tetraquis $(5,10,15,20$-2-nitrofeni)porfirinato]rutênio(II); complexo 2 = (carbonil)[tetraquis(5,10,15,20-2-nitrofenil- $\beta$ pentanitro)porfirinato]rutênio(II); complexo $3=$ (carbonil)[tetraquis(5,10,15,20-4-nitrofeni)porfirinato]rutênio(II) e complexo 4 = (carbonil)[tetraquis(5,10,15,20-4-nitrofenil- $\beta$-heptanitro)porfirinato]rutênio(II). 
duto para os complexos 2-nitrofenil-substituídos,o devido à provável distorção estrutural, provocada pelo efeito estéreo dos grupos nitro em posição 2-fenil interagindo com os grupos nitro da posição $\beta$-pirrólica. Isso faz com que o anel porfirínico distorça mais e o metal se desloque mais do centro desse anel, facilitando a aproximação do substrato e do doador de oxigênio ao centro metálico.

\section{AGRADECIMENTOS}

Os autores agradecem o apoio financeiro da FAPESP, CAPES e CNPq.

\section{REFERENNCIAS}

1. Hansen, C. B.; Agterberg, F. P. W.; van Eijndhoven, A. M. C.; Drenth W.; J. Mol. Catal. A: Chem. 1995, 102, 117.

2. Frauenkron, M.; Berkessel, A.; Tetrahedron Lett. 1997, 38, 7175.

3. Galardon, E.; Maux, P. L.; Simonneaux, G.; Tetrahedron 2000, 56, 615.

4. Gross, Z.; Ini, S.; Kapon, M.; Cohen, S.; Tetrahedron Lett. 1996, 40, 7325.

5. Vasil, V. V.; Borisov, S. M.; Sens. Actuators, B 2002, 82, 272.

6. Higuchi, T.; Hirobe, M.; J. Mol. Catal. A: Chem. 1996, 113, 403.

7. Groves, J. T.; Quinn, R.; J. Am. Chem. Soc. 1985, 107, 5790.

8. Groves, J. T.; Nemo, T. E.; Myers, R. S.; J. Am. Chem. Soc. 1979, 101, 1032.

9. Guo, C. C.; Huang, G.; Li, Z. P.; Song, J. X.; J. Mol. Catal. A: Chem. 2001, $170,43$.

10. Doro, F. G.; Lindsay, J. R.; Ferreira, A. G.; Assis, M. D.; J. Mol. Catal. A: Chem. 2000, 164, 97.

11. Haber, J.; Iwanejko, J.; Battioni, P.; Mansuy, D.; J. Mol. Catal. A: Chem. 2000, 152, 111.

12. Meunier, B.; Chem. Rev. 1992, 92, 1411.

13. Groves, J. T.; Quinn, R.; Inorg. Chem. 1994, 23, 3844.

14. Groves, J. T.; Ahn, K. H.; Inorg. Chem. 1987, 26, 3831.

15. Groves, J. T.; Shalyaev, K. V.; Bonchio, M.; Carofiglio, T.; $3^{\text {rd }}$ World Congress on Oxidation Catalysis, San Diego CA, USA, 1997.

16. Groves, J. T.; Roman, J. S.; J. Am. Chem. Soc. 1996, 118, 8961.

17. Mlodnicka, T.; James, B.R.; Em Metalloporphyrins catalyzed oxidations, Montanari, F.; Casella, L., eds., Kluwer Academic Publishers, 1994, p 121148.

18. Oka, Y. M.; Akita, M.; Catal. Today 1998, 41, 327.

19. Bressan, M.; Morvillo, A.; Inorg. Chem. 1989, 28, 950.

20. Chen, C. Y.; Cheng, S. H.; Su, Y. O.; J. Electroanal. Chem. 2000, 487, 51.
21. Morice, C.; Maux, P. L.; Moinet, C.; Simonneaux, G.; Inorg. Chim. Acta 1998, 273, 142 .

22. Hansen, C. B.; Agterberg, F. P. W.; Eijndhoven, A. M. C.; Drenth, W.; $J$ Mol. Catal. A: Chem. 1995, 102, 117.

23. Guimarães, C. A.; Dissertação de Mestrado, Universidade Federal de São Carlos, Brasil, 2002.

24. Leung, W. H.; Che, C. M.; J. Am. Chem. Soc. 1989, 111, 8812.

25. Cheng, S. Y. S.; James, B. R.; J. Mol. Catal. A: Chem. 1982, 117, 91.

26. Medforth, C. J.; Berber, M. D.; Smith, K. M.; Tetrahedron Lett. 1990, 31, 3719.

27. Barkigia, K. M.; Medforth, C. J.; Berber, M. D.; Fajer, J.; Renner, M. W.; Smith, K. M.; J. Am. Chem. Soc. 1990, 112, 8851.

28. Hombrecher, H. K.; Gherdan, V. M.; Ohm, S.; Cavaleiro, J. A. S.; Neves, M. G. P. M. S.; Condesso, M. F.; Tetrahedron 1993, 49, 8569.

29. Boschi, T.; D‘Arcangelo, G.; Tagliatesta, P.; J. Chem. Res., Synop. 1995, 326.

30. Lindsey, J. S.; Sheirman, I. C.; Kearney, P.C.; Marguerettaz, A. M.; J. Org. Chem. 1987, 52, 827.

31. Bartoli, J. F.; Battioni, P.; De Foor, W. R.; Mansuy, D.; Chem. Soc.: Chem. Commun. 1994, 23.

32. Collman, J. P.; Barnes, C. E.; Brothers, P. J.; Collins, T. J.; Ozawa, T.; Gallucci, J. C.; Ibers, J. A.; J. Am. Chem. Soc. 1984, 106, 5151.

33. Saltzman, H.; Sharefkin, J. G.; Baungratem, H. E., eds.; Organic Synthesis Collective, John Willey \& Sons: New York. 1973, vol. 5, p. 658.

34. Lucas, H. J.; Kennedy, E. R.; Formo, M. W.; Baungratem, H. E. eds.; Organic Synthesis Collective, John Willey \& Sons: New York. 1955, vol. 3, p. 483.

35. Bonnet, J. J.; Eaton, S. S.; Eaton, G. R.; Holm, R. H.; Ibers, J. A.; J. Am Chem. Soc. 1973, 4, 2141

36. Vitols, S. E.; Roman, J. S.; Ryan, D. E.; Blackwood Jr., M. E.; Spiro, T. G.; Inorg. Chem. 1997, 36, 764.

37. Spiro, T. G.; Hu, S.; J. Am. Chem. Soc. 1993, 115, 12029

38. Silverstein, R. M; Bassler, G. C.; Morrill, T. C.; Identificação Espectroscópica de Compostos Orgânicos, 5 ed., Guanabara Koogan, 1983, p. $85-152$.

39. Willis, H. A.; van der Maas, J. H.; Miller, R. G. J.; Laboratory Methods in Vibrational Spectroscopy, 3ed., John Willey \&Sons, 1972, p. 1-35.

40. Kanmani, A. S.; Vancheesan, S.; J. Mol. Catal. A: Chem. 1997, 125, 127.

41. Sheldon, R. A.; Barf, G. A.; J. Mol. Catal. A: Chem. 1995, 102, 23.

42. Ciuffi, K. J.; Tese de doutorado, Universidade Estadual Paulista, Brasil, 1997.

43. Schuchardt, U.; Cardoso, D.; Sercheli, R.; Pereira, R.; Cruz, R. S.; Guerreiro, M. C.; Mandelli, D.; Spinacé, E. V.; Pires, E. L.; Appl. Catal., A 2001, 211, 1 . 